\title{
Retrospective Cohort Analysis of the Reduced Burden of Hypoglycemia Associated with Dipeptidyl Peptidase-4 Inhibitor Use in Patients with Type 2 Diabetes Mellitus
}

\author{
Yuexin Tang · Jinan Liu · Hakima Hannachi · Samuel S. Engel • \\ Michael L. Ganz · Swapnil Rajpathak
}

Received: August 3, 2018 / Published online: October 4, 2018

(C) The Author(s) 2018

\begin{abstract}
Introduction: The use of antihyperglycemic agents (AHA), especially insulin and sulfonylureas (SU), is a risk factor for hypoglycemia. Despite the significant clinical and economic burdens associated with hypoglycemia and the decreasing use of SU in favor of other oral AHA, relatively little is known about hypoglycemia trends specific to the use of non-insulin AHA. We sought to estimate annual hypoglycemia event rates and costs among patients with type 2 diabetes mellitus (T2DM) who started either SU or dipeptidyl peptidase- 4 inhibitors (DPP-4i) and to predict rates and costs in the absence of DPP-4i.
\end{abstract}

Enhanced digital features To view enhanced digital features for this article go to https://doi.org/10.6084/ m9.figshare.7072649.

Electronic supplementary material The online version of this article (https://doi.org/10.1007/s13300018-0512-3) contains supplementary material, which is available to authorized users.

Y. Tang $(\bowtie) \cdot$ J. Liu $\cdot$ H. Hannachi ·

S. S. Engel · S. Rajpathak

Merck Research Laboratories Merck \& Co., Inc., Kenilworth, NJ, USA

e-mail: yuexin.tang@merck.com

M. L. Ganz

Evidera, Waltham, MA, USA

e-mail: michael.ganz@evidera.com
Methods: Truven's MarketScan Commercial Claims database was used to estimate hypoglycemia event rates and costs from 2007 to 2013. Hypoglycemia, defined using diagnosis codes, was assessed during the 12 months following SU $(n=245,201)$ or DPP-4i $(n=176,786)$ initiation by adults with T2DM. Coefficients from a Poisson regression model used to estimate the impact of patient characteristics on hypoglycemia rates for patients who started SU were used to predict rates for patients who started DPP-4i had they started SU instead.

Results: Hypoglycemia events per 100 patientyears (costs per event) ranged from $5.4(\$ 565)$ in 2007 to 10.4 ( $\$ 1154)$ in 2013 for patients starting SU; rates (costs) for patients starting DPP-4i ranged from $3.2(\$ 308)$ in 2007 to $6.4(\$ 482)$ in 2013. Predicted hypoglycemia rates would have been 5.3-9.9 per 100 person-years for patients who started DPP-4i had they started SU instead. Starting DPP-4i, rather than SU, would have resulted in national savings of $\$ 750.3$ million in healthcare costs due to avoided hypoglycemia events during this period.

Conclusions: Hypoglycemia rates and costs were consistently higher for patients who started SU rather than DPP-4i. The overall burden of hypoglycemia could be lowered substantially in the USA if, when feasible, patients with T2DM initiate DPP-4i instead of SU.

Funding: Merck \& Co., Inc., Kenilworth, NJ USA. 
Keywords: Dipeptidyl peptidase- 4 inhibitors; Hypoglycemia; Sulfonylureas; Type 2 diabetes mellitus

\section{INTRODUCTION}

Type 2 diabetes mellitus (T2DM) affects approximately 28 million individuals in the USA, a number that is expected to grow to almost 36 million by the year 2030 [1, 2]. Diabetes is responsible for $\$ 176$ billion in annual direct medical costs and $\$ 69$ billion annually in disability, lost work productivity, and premature mortality in the USA [3]. The goal of diabetes treatment is to achieve glycemic control and reduce the risk of long-term complications [4]. To be able to achieve and maintain their glycemic control goals and avoid complications, patients should remain adherent to their prescribed treatment regimen [5]. However, intensive glycemic control with use of antihyperglycemic agents (AHA), especially insulin and sulfonylureas (SU), could lead to increased risk of hypoglycemia [6-10], which is associated with decreased quality of life [11], higher risk of cardiovascular events, fractures, and death [12-17]. In addition, hypoglycemia episodes requiring third-party medical help or hospitalization can pose a significant economic burden to the healthcare system. The mean cost per hypoglycemic event treated on an outpatient basis ranges from $\$ 285$ to $\$ 394$ [2008 US dollars (USD)] [8, 18], while the mean cost per event treated on an inpatient basis ranges from $\$ 7562$ (2013 USD) [19] to $\$ 17,564$ (2008 USD) [18]. Zhao et al. [33] have estimated that hypoglycemia is responsible for anywhere from $\$ 1.8$ to $\$ 3.5$ billion (2009 USD) per year in the USA in direct medical costs.

Several personal and clinical factors are related to patients' willingness and ability to adhere to their treatment regimens, with fear of hypoglycemia being one of the most important barriers to adherence [20-23]. Some diabetes medications are associated with higher rates of hypoglycemia than others. For example, Lipska et al. examined medical and pharmacy claims from 2006 through 2013 and reported that incidence rates for severe hypoglycemia resulting in a hospital admission, observation stay, or emergency department (ED) visit were about four times higher for patients using insulin or SU than for patients using other AHA (1.80-2.0 vs. 0.2 per 100 patient-years) [24]. The period they studied coincided with one during which diabetes treatment patterns changed. For example, dipeptidyl peptidase-4 inhibitors (DPP-4i), which were first approved by the US Food and Drug Administration in 2006 and entered the US market in October of that year, have been shown to be associated with lower risks of hypoglycemia and hospitalization for hypoglycemia than SU [25-27].

Despite the significant clinical and economic burdens associated with hypoglycemia and the decreasing use of SU in favor of other oral AHA [28], relatively little literature is devoted to hypoglycemia trends specific to the use of noninsulin AHA in recent years. The objectives of this study are, therefore, to estimate annual hypoglycemia event rates and costs among patients with T2DM who have started either SU or DPP-4i, and to predict hypoglycemia event rates and costs in the absence of DPP- $4 \mathrm{i}$ from 2007 to 2013.

\section{METHODS}

\section{Data Source, Study Design, and Study Sample Selection}

We analyzed encounters and services that occurred from January 2007 to December 2013 using data from the Truven MarketScan Commercial Claims and Encounters database. Since 1995, this database have accumulated health insurance enrollment and claims information for approximately 185 million commercially insured working age adults and their dependents. Enrollment data, medical claims, and pharmacy claims are linked to each patient using an encrypted identification number.

We selected patients 18-64 years old with evidence of T2DM who were newly treated with SU or DPP-4i during each year and who were continuously enrolled in their health plan with medical and pharmacy benefits for at least 12 months prior to starting their index therapy 

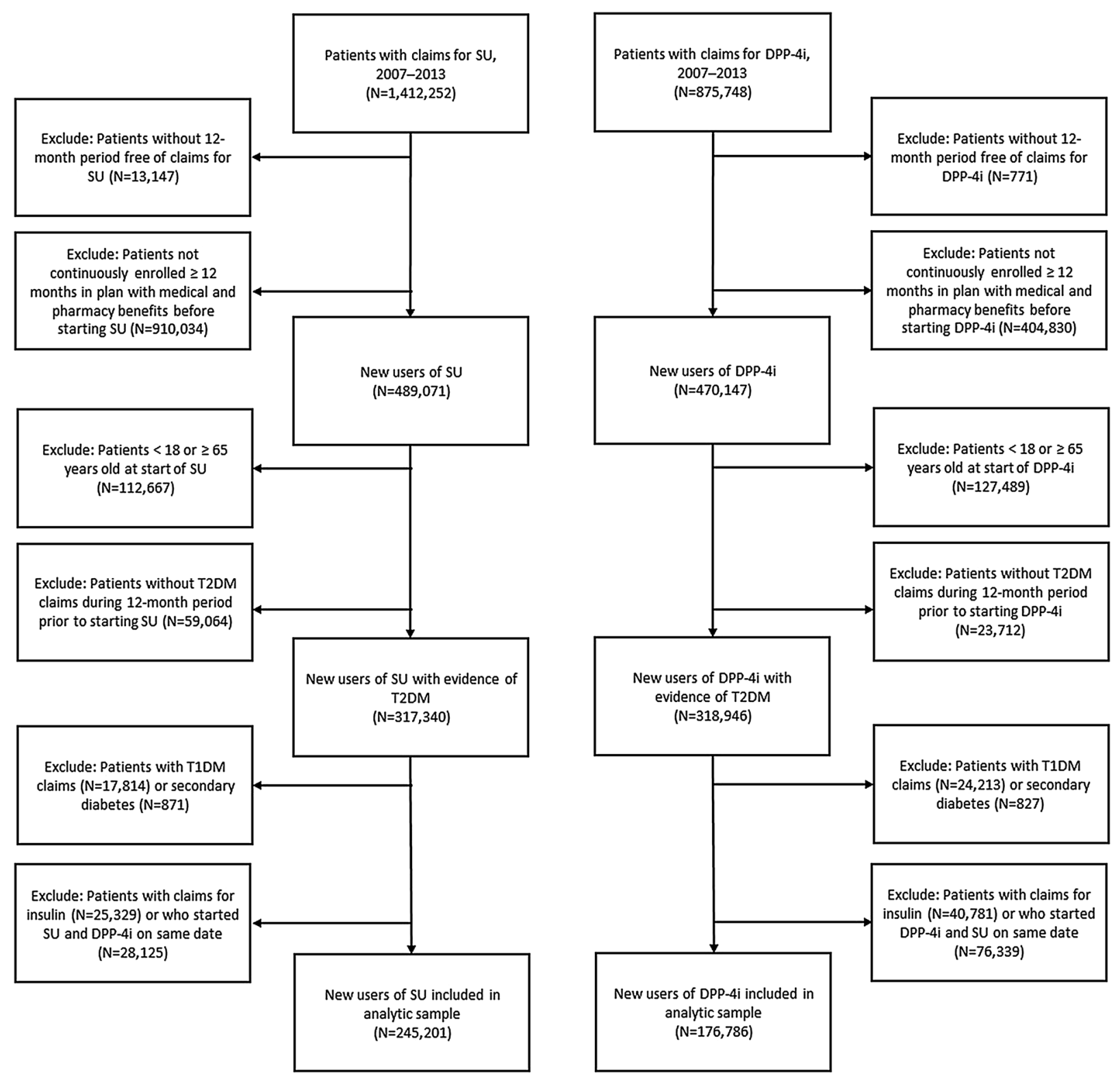

Fig. 1 Sample attrition flowchart. DPP-4i dipeptidyl peptidase-4 inhibitors, $S U$ sulfonylureas, TIDM type 1 diabetes mellitus, T2DM type 2 diabetes mellitus

(SU or DPP-4i) (Fig. 1). Specifically, patients were included if they had at least one claim during the 12 months before they started their index therapy (baseline period) with a T2DM diagnosis code (International Classification of Diseases, Ninth Revision, Clinical Modification [ICD-9-CM] code 250.x0 or 250.x2) and who did not have a claim for their index therapy during the baseline period. Patients were excluded from the sample if they had any claims with type 1 diabetes mellitus codes (ICD-9-CM code $250 . x 1$ or $250 . x 3$ ), had any evidence of secondary diabetes (ICD-9-CM codes 249.xx), had a prescription for insulin during the baseline period, or initiated SU and DPP-4i on the same date. Patients were followed from the date they started their index therapy until the last date of continuous enrollment in medical and pharmacy benefits, the end of the index therapy, or start of insulin, whichever came first. 
This article is based on previously collected data and does not report any results based on studies of human participants performed by any of the authors.

\section{Study Measures}

\section{Demographic and Clinical Characteristics}

Patients' demographic and clinical characteristics included age; gender; geographic region of residence; presence of comorbid conditions, including cardiovascular disease; and use of antihyperglycemic, antihypertensive, and antihyperlipidemic medications. Demographic characteristics were assessed as of the first day of the follow-up period; any presence of comorbid conditions and use of medications were assessed during the baseline period. A complete list of the comorbid conditions, and their definitions, is available in Appendix A. In addition to measuring baseline health status using individual health conditions, we also computed a version of the Charlson Comorbidity Index (CCI) modified by Quan et al. [29].

\section{Hypoglycemia Events and Costs}

Hypoglycemia events were defined as those outpatient, inpatient, or ED services with a hypoglycemia-related diagnosis that occurred during the follow-up period. Claims with hypoglycemia diagnosis codes that occurred on consecutive days were considered part of the same single event unless interrupted by at least 1 day without evidence of hypoglycemia, in which case, each run of consecutive days of with hypoglycemia diagnosis codes was considered a separate event (hypoglycemia events occurring in the outpatient and inpatient/ED settings on the same day were considered inpatient/ED events). Consistent with previously published research, we identified hypoglycemia events by the presence of diagnosis codes 251.0, 251.1, 251.2, 270.3, or 962.3; or the presence of diagnosis codes $251.8 \mathrm{x}$ without codes 259.8, 272.7, 681.xx, 682.xx, 686.9, 707.1x. 707.2x, 707.8, 707.9, 709.3, 730.1x, $730.2 \mathrm{x}$, or 731.8 in the ICD-9-CM diagnosis fields [30]. The original algorithm for identifying hypoglycemia-related events published by
Ginde et al. [30] included ICD-9-CD codes 270.3 (leucine-induced hypoglycemia), 775.0 (hypoglycemia in an infant born to a diabetic mother), and 775.6 (neonatal hypoglycemia), which we excluded as a result of the sample selection criteria.

Online Appendix B describes in detail the multistage process we followed to predict hypoglycemia rates. We developed and validated a Poisson regression model to estimate the impact of patient characteristics, adjusted for differences in follow-up duration, on hypoglycemia events experienced by patients who started SU. The estimated model was then used to predict hypoglycemia events experienced by patients who started DPP-4i had they started SU instead. The difference between the observed and predicted rates represents the hypoglycemia burden associated with starting DPP-4i instead of SU.

Hypoglycemia-related costs were measured by payments for hypoglycemia-related services, defined above. The costs for all claims related to the same hypoglycemia event were summed and expressed in nominal 2013 dollars using the medical care component of the Consumer Price Index. To estimate observed and predicted aggregate hypoglycemia costs for the entire USA, we combined the average costs per hypoglycemia event experienced by patients who have started DPP-4i and SU with the observed and predicted hypoglycemia rates and the number of patients using DPP-4i in the USA derived from the National Health and Nutrition Examination Survey. The difference between the observed and predicted aggregate hypoglycemia costs for patients who started DPP-4i estimates the hypoglycemia-related healthcare costs saved, on the national level, associated with starting DPP-4i instead of SU (see Online Appendix B for details).

\section{Statistical Analyses}

Patient baseline demographic and clinical characteristics were summarized by their means and standard deviations for continuous measures and their proportions for categorical variables. Crude annual hypoglycemia event rates 
were calculated as the total number of events divided by the total number of patient-years, defined as the number of days divided by 365 , observed during the follow-up period. The 95\% confidence intervals (CI) for the hypoglycemia event rates were obtained using the Poisson distribution. Patient characteristics, observed hypoglycemia rates, and costs were stratified by index therapy; predicted hypoglycemia rates and costs were computed for patients using DPP-4i.

\section{RESULTS}

\section{Patient Characteristics}

We identified 245,201 and 176,786 patients newly treated with SU and DPP-4i, respectively. Patients in both groups were 52-53 years old and $44-46 \%$ were women. Patients in both groups generally had similar distributions of comorbid conditions, including cardiovascular disease (Table 1). Patients in the SU group were less likely than patients in the DPP-4i group to have hypertension (53\% vs. 58\%) and hyperlipidemia ( $48 \%$ vs. $57 \%$ ). They were also less likely to use metformin (61\% vs. $69 \%$ ), thiazolidinediones (13\% vs. $25 \%)$, antihypertensive medications (59\% vs. $65 \%$ ), and antihyperlipidemic medications (48\% vs. 59\%) at baseline. Almost $5 \%$ of patients in the SU group were already using DPP-4i before starting SU therapy and $17 \%$ of patients in the DPP- 4 i group were already using SU before starting DPP-4i therapy.

\section{Hypoglycemia Event Rates and Costs}

Annual hypoglycemia event rates were consistently higher for patients who started using SU than DPP-4i (Table 2; see Appendix C for 95\% CI). Patients who started SU experienced 5.4 hypoglycemia events per 100 patient-years in 2007 to 10.4 per 100 patient-years in 2013, while patients who started DPP-4i experienced 3.2 hypoglycemia events per 100 patient-years in 2007 to 6.4 per 100 patient-years in 2013. As shown in Appendix C, the increasing trends in hypoglycemia event rates reflect the patterns in outpatient hypoglycemic event rates; inpatient/ ED event rates, and the proportion of events due to inpatient/ED events, remained relatively unchanged during the 7 years. In addition to higher rates, patients who started using SU also experienced more expensive events. Costs of hypoglycemia events ranged, on average, from $\$ 565$ in 2007 to $\$ 1154$ in 2013 for patients who started SU and from \$308 in 2007 to \$482 in 2013 for patients who started DPP-4i.

Our prediction model indicated that patients in the DPP-4i group would have experienced more hypoglycemia events had they started using SU instead (5.3 per 100 patient-years in 2007 to 9.9 per 100 patient-years in 2013), close to the levels observed for patients who started using SU (Fig. 2). In other words, the use of DPP$4 \mathrm{i}$ as the index therapy, rather than $\mathrm{SU}$, was associated with event rates that were 35\% (in 2013 ) to $57 \%$ (in 2009), for an average of $46 \%$, lower from 2007 to 2013. Figure 3 displays the annual total observed and predicted costs of hypoglycemia events for patients who started using DPP-4i. Total observed hypoglycemia-related costs ranged from $\$ 9.6$ million in 2007 to $\$ 73.7$ million in 2013 , for a total $\$ 264.3$ million over 7 years. Total predicted costs, had the patient started SU instead of DPP-4i, ranged from \$29.1 million in 2007 to $\$ 273.1$ million in 2013 , for a total of $\$ 1014.9$ million over 7 years. Starting treatment with DPP-4i, rather than SU, resulted in savings of $\$ 750.3$ million in healthcare costs over 7 years due to avoided hypoglycemia events (see Online Appendix B for further details).

\section{DISCUSSION}

Past literature has presented mixed information about national hypoglycemia trends in the USA. For example, although Lipska et al. reported that annual rates of hospitalization for hypoglycemia among Medicare fee-for-service beneficiaries have increased approximately $12 \%$ between 1999 and 2011 [31], other researchers have reported conflicting data. Wang et al. [32], using national survey data, found that hypoglycemia-related ED visit rates decreased $22 \%$ from 1.8 (in 2006) to 1.4 (in 2011) per 100 
Table 1 Baseline demographic and clinical characteristics of patients starting sulfonylureas and dipeptidyl peptidase- 4 inhibitors

\begin{tabular}{|c|c|c|}
\hline Characteristic & Sulfonylureas $(N=245,201)$ & $\begin{array}{l}\text { Dipeptidyl peptidase- } 4 \\
\text { inhibitors }(N=176,786)\end{array}$ \\
\hline Mean (SD) age, years & $51.9(8.9)$ & $52.8(8.3)$ \\
\hline Female, \% & 43.5 & 45.5 \\
\hline \multicolumn{3}{|l|}{ Geographic region, \% } \\
\hline Northeast & 9.9 & 12.1 \\
\hline Midwest & 24.1 & 22.3 \\
\hline West & 47.6 & 52.1 \\
\hline South & 17.6 & 12.8 \\
\hline Mean (SD) Charlson comorbidity index & $1.5(1.3)$ & $1.6(1.2)$ \\
\hline \multicolumn{3}{|l|}{ Comorbid conditions, $\%$} \\
\hline Hypertension & 53.3 & 57.5 \\
\hline Hyperlipidemia & 48.4 & 56.5 \\
\hline Obesity or overweight & 9.1 & 8.6 \\
\hline Cataracts, macular edema, retinopathy, or blindness & 8.0 & 10.0 \\
\hline Neuropathy & 7.4 & 8.5 \\
\hline Depression & 6.5 & 6.3 \\
\hline Chronic obstructive pulmonary disease & 5.5 & 5.0 \\
\hline Cancer & 4.3 & 4.3 \\
\hline Chronic kidney diseases or nephropathy & 3.8 & 4.0 \\
\hline Hypoglycemia & 2.3 & 2.0 \\
\hline Proteinuria & 2.2 & 2.4 \\
\hline Fracture & 1.9 & 1.9 \\
\hline Sleep disorder & 1.6 & 1.8 \\
\hline HIV/AIDS & 0.2 & 0.2 \\
\hline Alzheimer's disease, dementia, or MCI & 0.1 & 0.1 \\
\hline \multicolumn{3}{|l|}{ Cardiovascular disease, $\%$} \\
\hline Arrhythmia & 4.5 & 4.4 \\
\hline Cerebrovascular disease & 3.2 & 3.4 \\
\hline Congestive heart failure & 3.1 & 2.8 \\
\hline Peripheral arterial disease & 2.7 & 3.2 \\
\hline Stroke/transient ischemic attacks & 2.6 & 2.8 \\
\hline Myocardial infarction & 1.7 & 1.3 \\
\hline
\end{tabular}


Table 1 continued

\begin{tabular}{lcc}
\hline Characteristic & Sulfonylureas $(\boldsymbol{N}=\mathbf{2 4 5 , 2 0 1})$ & $\begin{array}{c}\text { Dipeptidyl peptidase-4 } \\
\text { inhibitors }(\boldsymbol{N}=\mathbf{1 7 6 , 7 8 6})\end{array}$ \\
\hline Angina pectoris & 1.6 & 1.8 \\
Use of antihyperglycemic medications, \% & 68.8 \\
Metformin & 61.1 & 25.1 \\
Thiazolidinediones & 13.1 & 0.0 \\
Dipeptidyl peptidase-4 inhibitors & 4.8 & 1.9 \\
Meglitinides & 0.9 & 0.3 \\
Alpha-glucosidase inhibitors & 0.1 & 16.9 \\
Sulfonylureas & 0.0 & 65.3 \\
Use of antihypertensive medications, \% & 58.5 & 58.9 \\
Use of antihyperlipidemic medications, \% & 47.8 & \\
\hline
\end{tabular}

$A I D S$ acquired immune deficiency syndrome, $H I V$ human immunodeficiency syndrome, $M C I$ mild cognitive impairment, $S D$ standard deviation

Table 2 Hypoglycemia event rates and costs among patients starting sulfonylureas and dipeptidyl peptidase-4 inhibitors

\begin{tabular}{|c|c|c|c|c|c|c|c|}
\hline & 2007 & 2008 & 2009 & 2010 & 2011 & 2012 & 2013 \\
\hline \multicolumn{8}{|c|}{ Hypoglycemia event rate per 100 patient-years } \\
\hline Sulfonylureas & 5.4 & 6.7 & 8.1 & 8.2 & 7.5 & 9.0 & 10.4 \\
\hline Dipeptidyl peptidase- 4 inhibitors & 3.2 & 3.0 & 3.2 & 3.6 & 4.3 & 4.3 & 6.4 \\
\hline \multicolumn{8}{|l|}{ Mean cost per hypoglycemia event, $\$$} \\
\hline Sulfonylureas & 565 & 1168 & 1035 & 880 & 931 & 1098 & 1154 \\
\hline Dipeptidyl peptidase- 4 inhibitors & 308 & 514 & 915 & 410 & 365 & 477 & 482 \\
\hline
\end{tabular}

adults and Zhao et al. [33], also using national survey data, found that there were almost 100,000 fewer hospital admissions from ED visits in 2009 than in 2005. Most recently, using data from private health insurance and Medicare Advantage claims and a network of 11 integrated health systems, neither Pathak et al. [34] nor Lipska et al. [24] found any significant upward or downward changes in severe hypoglycemia rates between 2005-2011 and 2006-2013, respectively, even though diabetes treatment patterns changed substantially in the same period [24, 34-37].
This study contributes to the literature on the burden of hypoglycemia-related events by providing more recent data and by focusing on the differences between two oral AHA of different classes and vintages. While we found that overall, inpatient/ED and outpatient hypoglycemia event rates associated with starting SU or DPP-4i increased over time, which is consistent with data on the increasing proportion of adults using antidiabetic agents [38], we also found that hypoglycemia event rates associated with SU were consistently higher and grew at a slightly faster pace than rates associated with DPP-4i. We also found that for patients who 


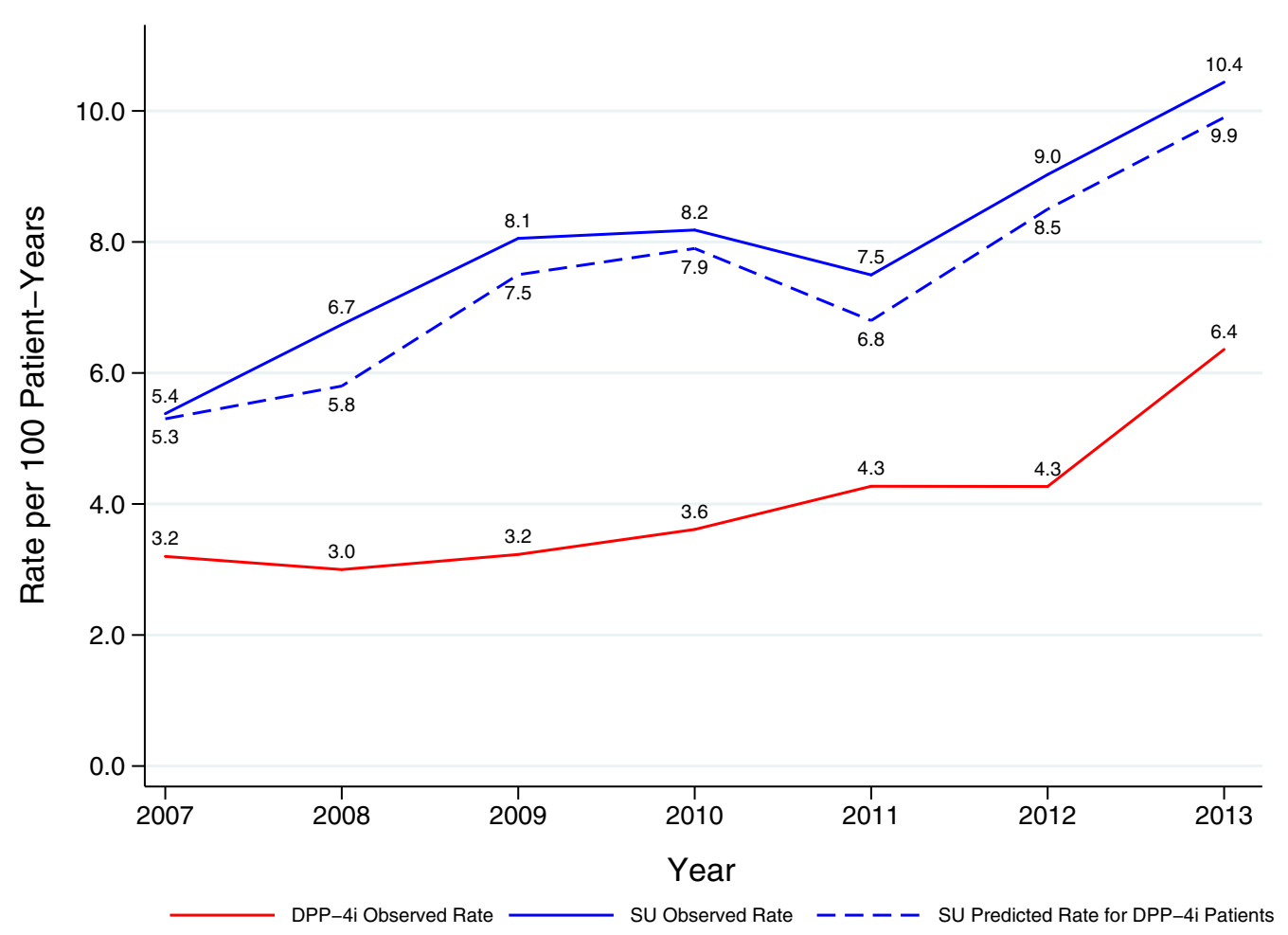

Fig. 2 Hypoglycemia event rates among patients starting sulfonylureas and dipeptidyl peptidase-4 inhibitors. DPP-4i dipeptidyl peptidase-4 inhibitors, $S U$ sulfonylureas

started SU, inpatient/ED hypoglycemic event rates were a smaller portion of their total event rates than for patients who started DPP-4i and that hypoglycemia-related events associated with starting SU were also consistently more expensive than events associated with starting DPP-4i. Furthermore, we found, through our predictive model, that starting DPP-4i, instead of SU, from 2007 to 2013 was associated with nearly 50\% fewer (i.e., avoided) hypoglycemia events, which corresponded to a total savings in hypoglycemia-related healthcare costs of $\$ 750$ million over 7 years.

\section{Limitations}

Using claims and other administrative data to study hypoglycemia-related healthcare events is challenging. Although it is unlikely that hypoglycemia-related services were incorrectly identified as such in the claims data we analyzed, it is possible that some hypoglycemia events were not captured or recorded correctly, particularly if the events did not result in a billable service, did not involve an interaction with a healthcare provider, or an appropriate diagnosis code was not used, which could be quite common [39]. The degree to which these cost estimates are biased may also vary with patient characteristics if, as Veronese et al. have shown using Italian data [40], underreporting is related to disease severity. The data we analyzed only captured hypoglycemia events that required medical care or hospitalization; as a result, hypoglycemia burden may be underestimated. It could be argued that since we observed hypoglycemia-related events that involved interactions with healthcare providers and facilities, these hypoglycemia events were likely to be severe; however, no evidence about their severity is available. We were unable to differentiate between daytime and nocturnal hypoglycemia nor could we account for several clinical characteristics known to be associated with hypoglycemia such as HbA1c, body mass index, renal function, and lipid levels that are not typically available in claims data. We were also unable to 


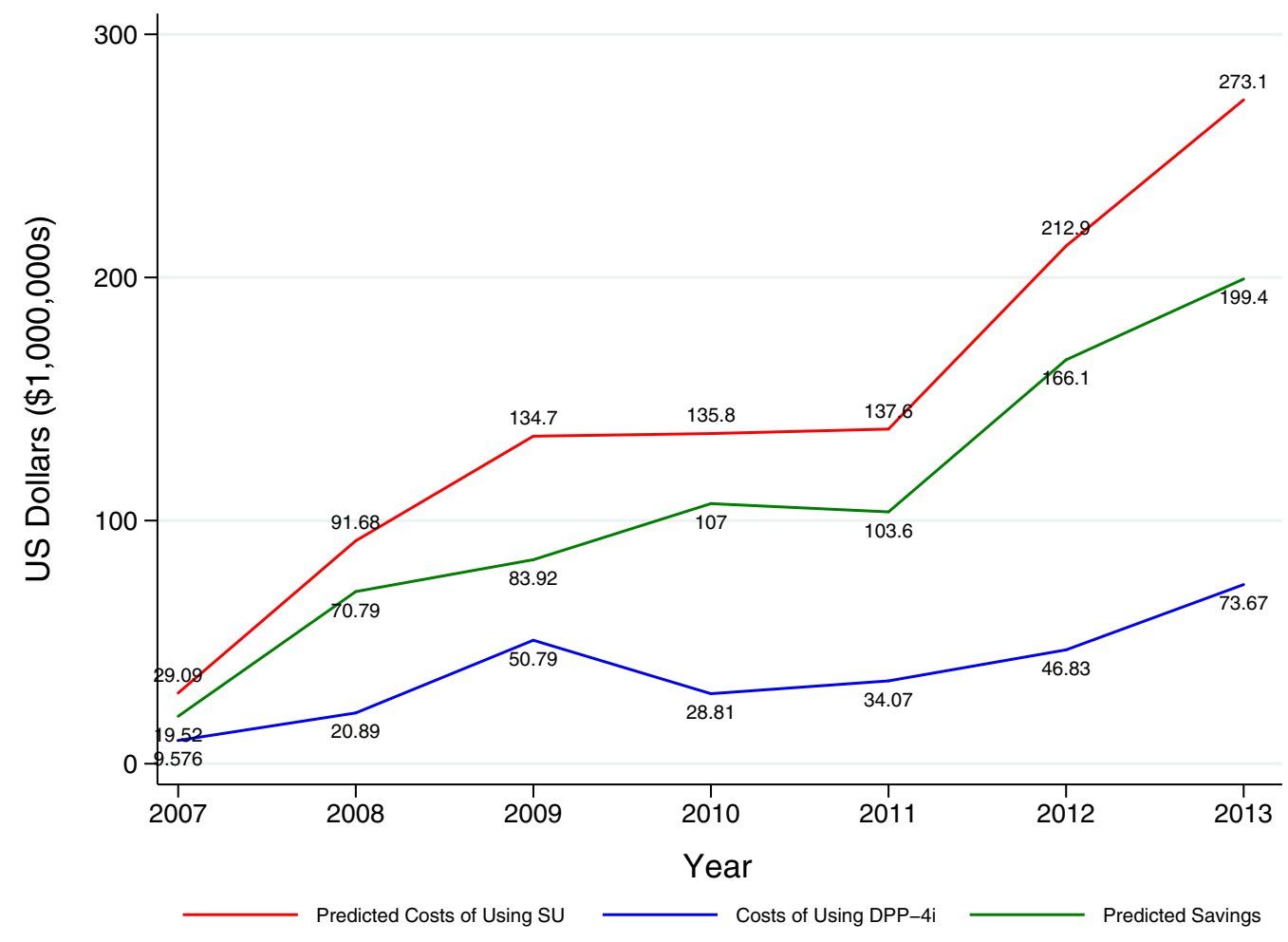

Fig. 3 Direct cost savings related to reduced hypoglycemia burden among patients starting dipeptidyl peptidase-4 inhibitors (2013 US dollars). DPP-4i dipeptidyl peptidase-4 inhibitors, $S U$ sulfonylureas

account or adjust for duration of diabetes, or events and costs beyond 12 months from patients' start of their index therapies. Our exclusion criteria may also have resulted in a sample that was healthier (no inulin use prior to start of SU or DPP-4i) than the target population of T2DM patients using these regimens. Because we focused on hypoglycemia-related costs, other aspects of the economic burden related to use of these medications, such as medication costs or the economic implications of weight changes, were not considered in this study. Furthermore, our results are based on commercially insured patients 18-64 years old and retirees covered by supplemental Medicare plans who were followed for no more than 12 months. Caution, therefore, is recommended when generalizing these findings to other populations.

\section{CONCLUSIONS}

Despite these limitations, our results for hypoglycemia rates are consistent with recent meta- analyses of population-based studies and clinical trials that compared hypoglycemia rates across multiple treatments for T2DM. Edridge et al. reported that 5\% (for severe) to 30\% (for mild/moderate) of patients using SU experienced a hypoglycemia event [35] while Mishriky et al. reported that $20 \%$ and $6 \%$ of patients using SU and DPP-4i, respectively, experienced an incident hypoglycemia event since starting those agents [36]. Mearns et al. found, on the basis of a network meta-analysis of 62 clinical trials involving 25 agents, that the risk of confirmed hypoglycemia among patients using SU was 3.1-71.3 (3.1-25.3 excluding alogliptin) times higher than for patients using DPP-4i [37].

In addition to confirming what has been reported in the recent literature, our results also provide comparative information on the annual rates and average costs of hypoglycemia events since DPP-4i became available in the USA: hypoglycemia rates and costs associated with starting SU are higher than those associated with DPP-4i. This information, to our 
knowledge, has not been previously published. Data from our predictive model also show that the overall burden of hypoglycemia could be lowered substantially if, when clinically feasible, patients with T2DM started using DPP-4i instead of SU.

\section{ACKNOWLEDGEMENTS}

Funding. This study and resulting manuscript article processing charges were funded by Merck \& Co., Inc., Kenilworth, NJ USA. All authors had full access to all of the data in this study and take complete responsibility for the integrity of the data and accuracy of the data analysis.

Editorial Assistance. The authors would like to acknowledge the contribution of Kivilcim Sungur-Stasik of Merck \& Co., Inc., Kenilworth, $\mathrm{NJ}$, USA for her assistance in preparing this manuscript.

Authorship. All named authors meet the International Committee of Medical Journal Editors (ICMJE) criteria for authorship for this article, take responsibility for the integrity of the work as a whole, and have given their approval for this version to be published.

Authorship Contributions. Yuexin Tang, Hakima Hannachi, Samuel S. Engel, and Swapnil Rajpathak conceived and designed the study, interpreted the data, and revised the manuscript. Jinan Liu designed the study, analyzed and interpreted the data, and revised the manuscript. Michael L. Ganz interpreted the data and drafted and revised the manuscript.

Prior Presentations. Some of the study findings have been presented at the American Diabetes Association's 77th Scientific Sessions (June 2017) and the 53rd Annual Meeting of the European Association for the Study of Diabetes (September 2017).
Disclosures. Yuexin Tang is an employee and shareholder of Merck \& Co., Inc., Kenilworth, NJ, USA. Jinan Liu was an employee of Merck \& Co., Inc., Kenilworth, NJ, USA at the time of this study. Hakima Hannachi is an employee and shareholder of Merck \& Co., Inc., Kenilworth, NJ, USA. Samuel S. Engel is an employee and shareholder of Merck \& Co., Inc., Kenilworth, NJ, USA. Michael L. Ganz is an employee of Evidera, which provides consulting and other research services to pharmaceutical, medical device, and other related businesses. In his salaried position, he works with a variety of companies and clients and is precluded from receiving payment or honoraria directly from these organizations for services rendered. Swapnil Rajpathak is an employee and shareholder of Merck \& Co., Inc., Kenilworth, NJ, USA.

Compliance with Ethics Guidelines. This article is based on previously collected data and does not report any results based on studies of human participants performed by any of the authors.

Data Availability. The claims data used for this study are available, via licensing agreements, from Truven Health Analytics. The computer code used to create the analytic dataset and perform the analyses is available from the corresponding author on reasonable request.

Open Access. This article is distributed under the terms of the Creative Commons Attribution-NonCommercial 4.0 International License (http://creativecommons.org/licenses/ by-nc/4.0/), which permits any noncommercial use, distribution, and reproduction in any medium, provided you give appropriate credit to the original author(s) and the source, provide a link to the Creative Commons license, and indicate if changes were made. 


\section{REFERENCES}

1. American Diabetes Association. Statistics about diabetes. 2016; http://www.diabetes.org/diabetesbasics/statistics/. Accessed 2 Dec 2016.

2. Shaw JE, Sicree RA, Zimmet PZ. Global estimates of the prevalence of diabetes for 2010 and 2030. Diabetes Res Clin Pract. 2010;87(1):4-14.

3. American Diabetes A. Economic costs of diabetes in the U.S. in 2012. Diabetes Care. 2013;36(4):1033-46.

4. American Diabetes Association. Glycemic targets. Sec. 6 in standards of medical care in diabetes2015. Diabetes Care. 2015;38(Suppl 1):S33-40.

5. Garcia-Perez LE, Alvarez M, Dilla T, Gil-Guillen V, Orozco-Beltran D. Adherence to therapies in patients with type 2 diabetes. Diabetes Ther. 2013;4(2):175-94.

6. Bodmer M, Meier C, Krahenbuhl S, Jick SS, Meier CR. Metformin, sulfonylureas, or other antidiabetes drugs and the risk of lactic acidosis or hypoglycemia: a nested case-control analysis. Diabetes Care. 2008;31(11):2086-91.

7. Cryer PE. Glycemic goals in diabetes: trade-off between glycemic control and iatrogenic hypoglycemia. Diabetes. 2014;63(7):2188-95.

8. Curkendall S, Zhang B, Oh K, Williams S, Pollack M, Graham J. Incidence and cost of hypoglycemia among patients with type 2 diabetes in the United States: analysis of a health insurance database. J Clin Outcomes Manag. 2011;18(10):455-62.

9. Karter AJ, Warton EM, Lipska KJ, et al. Development and validation of a tool to identify patients with type 2 diabetes at high risk of hypoglycemia-related emergency department or hospital use. JAMA Intern Med. 2017;177(10):1461-70.

10. Roumie CL, Min JY, Greevy RA, et al. Risk of hypoglycemia following intensification of metformin treatment with insulin versus sulfonylurea. CMAJ. 2016;188(6):E104-12.

11. Simon D, de Pablos-Velasco P, Parhofer KG, et al. Hypoglycaemic episodes in patients with type 2 diabetes-risk factors and associations with patientreported outcomes: the PANORAMA Study. Diabetes Metab. 2015;41(6):470-9.

12. Bonds DE, Miller ME, Bergenstal RM, et al. The association between symptomatic, severe hypoglycaemia and mortality in type 2 diabetes: retrospective epidemiological analysis of the ACCORD study. BMJ. 2010;340:b4909.
13. Hsu PF, Sung SH, Cheng HM, et al. Association of clinical symptomatic hypoglycemia with cardiovascular events and total mortality in type 2 diabetes: a nationwide population-based study. Diabetes Care. 2013;36(4):894-900.

14. Johnston SS, Conner C, Aagren M, Ruiz K, Bouchard J. Association between hypoglycaemic events and fall-related fractures in Medicare-covered patients with type 2 diabetes. Diabetes Obes Metab. 2012;14(7):634-43.

15. Johnston SS, Conner C, Aagren M, Smith DM, Bouchard J, Brett J. Evidence linking hypoglycemic events to an increased risk of acute cardiovascular events in patients with type 2 diabetes. Diabetes Care. 2011;34(5):1164-70.

16. McCoy RG, Van Houten HK, Ziegenfuss JY, Shah $\mathrm{ND}$, Wermers RA, Smith SA. Increased mortality of patients with diabetes reporting severe hypoglycemia. Diabetes Care. 2012;35(9):1897-901.

17. Zoungas S, Patel A, Chalmers J, et al. Severe hypoglycemia and risks of vascular events and death. N Engl J Med. 2010;363(15):1410-8.

18. Quilliam BJ, Simeone JC, Ozbay AB, Kogut SJ. The incidence and costs of hypoglycemia in type 2 diabetes. Am J Manag Care. 2011;17(10):673-80.

19. Candrilli SD, Meyers JL, Boye K, Bae JP. Health care resource utilization and costs during episodes of care for type 2 diabetes mellitus-related comorbidities. J Diabetes Complicat. 2015;29(4):529-33.

20. Brod M, Rana A, Barnett AH. Impact of self-treated hypoglycaemia in type 2 diabetes: a multinational survey in patients and physicians. Curr Med Res Opin. 2012;28(12):1947-58.

21. Fidler C, Elmelund Christensen T, Gillard S. Hypoglycemia: an overview of fear of hypoglycemia, quality-of-life, and impact on costs. J Med Econ. 2011;14(5):646-55.

22. International Hypoglycaemia Study Group. Minimizing hypoglycemia in diabetes. Diabetes Care. 2015;38(8):1583-91.

23. Wild D, von Maltzahn R, Brohan E, Christensen T, Clauson P, Gonder-Frederick L. A critical review of the literature on fear of hypoglycemia in diabetes: implications for diabetes management and patient education. Patient Educ Couns. 2007;68(1):10-5.

24. Lipska KJ, Yao X, Herrin J, et al. Trends in drug utilization, glycemic control, and rates of severe hypoglycemia, 2006-2013. Diabetes Care. 2017;40(4):468-75. 
25. Ahren B. Are sulfonylureas less desirable than DPP-4 inhibitors as add-on to metformin in the treatment of type 2 diabetes? Curr Diab Rep. 2011;11(2): 83-90.

26. Detournay B, Halimi S, Robert J, Deschaseaux C, Dejager S. Hypoglycemia hospitalization frequency in patients with type 2 diabetes mellitus: a comparison of dipeptidyl peptidase 4 inhibitors and insulin secretagogues using the French health insurance database. Vasc Health Risk Manag. 2015;11:417-25.

27. Raju A, Shetty S, Cai B, D'Souza AO. Hypoglycemia incidence rates and associated health care costs in patients with type 2 diabetes mellitus treated with second-line linagliptin or sulfonylurea after metformin monotherapy. J Manag Care Spec Pharm. 2016;22(5):483-92.

28. Turner LW, Nartey D, Stafford RS, Singh S, Alexander GC. Ambulatory treatment of type 2 diabetes in the U.S., 1997-2012. Diabetes Care. 2014;37(4):985-92.

29. Quan H, Sundararajan V, Halfon P, et al. Coding algorithms for defining comorbidities in ICD-9-CM and ICD-10 administrative data. Med Care. 2005;43(11):1130-9.

30. Ginde AA, Blanc PG, Lieberman RM, Camargo CA Jr. Validation of ICD-9-CM coding algorithm for improved identification of hypoglycemia visits. BMC Endocr Disord. 2008;8:4.

31. Lipska KJ, Ross JS, Wang Y, et al. National trends in US hospital admissions for hyperglycemia and hypoglycemia among Medicare beneficiaries, 1999 to 2011. JAMA Intern Med. 2014;174(7):1116-24.

32. Wang J, Geiss L, Williams D, Gregg E. Trends in emergency department visit rates for hypoglycemia and hyperglycemic crisis among adults with diabetes, United States, 2006-2011. PLoS One. 2015;10(8):e0134917.

33. Zhao Y, Shi Q, Wang Y, Fonseca V, Shi L. Economic burden of hypoglycemia: utilization of emergency department and outpatient services in the United States (2005-2009). J Med Econ. 2016;19(9):852-7.

34. Pathak RD, Schroeder EB, Seaquist ER, et al. Severe hypoglycemia requiring medical intervention in a large cohort of adults with diabetes receiving care in U.S. integrated health care delivery systems: 2005-2011. Diabetes Care. 2016;39(3):363-70.

35. Edridge CL, Dunkley AJ, Bodicoat DH, et al. Prevalence and incidence of hypoglycaemia in 532,542 people with type 2 diabetes on oral therapies and insulin: a systematic review and meta-analysis of population based studies. PLoS One. 2015;10(6): e0126427.

36. Mishriky BM, Cummings DM, Tanenberg RJ. The efficacy and safety of DPP4 inhibitors compared to sulfonylureas as add-on therapy to metformin in patients with type 2 diabetes: a systematic review and meta-analysis. Diabetes Res Clin Pract. 2015;109(2):378-88.

37. Mearns ES, Sobieraj DM, White CM, et al. Comparative efficacy and safety of antidiabetic drug regimens added to metformin monotherapy in patients with type 2 diabetes: a network metaanalysis. PLoS One. 2015;10(4):e0125879.

38. National Center for Health Statistics. Health, United States, 2016: with chartbook on long-term trends in health. Hyattsville, MD. 2017. https:// www.cdc.gov/nchs/data/hus/hus16.pdf. Accessed 8 Jan 2018.

39. Jakubczyk M, Rdzanek E, Niewada M, Czech M. Economic resources consumption structure in severe hypoglycemia episodes: a systematic review and meta-analysis. Expert Rev Pharmacoecon Outcomes Res. 2015;15(5):813-22.

40. Veronese G, Marchesini G, Forlani G, Fabbri A, Italian Society of Emergency Medicine (SIMEU). Are severe hypoglycemic episodes in diabetes correctly identified by administrative data? Evidence of underreporting from the HYPOTHESIS study. Acta Diabetol. 2016;53(4):677-80. 